\title{
Clinical study of acute toxicity of pelvic bone marrow-sparing intensity-modulated radiotherapy for cervical cancer
}

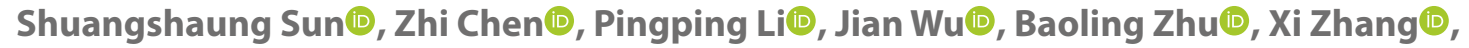

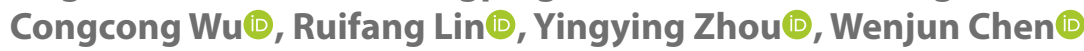 \\ Wenzhou Medical University, China
}

\begin{abstract}
Objectives: To compare the dose volume of the target area and the toxicity of pelvic bone marrow-sparing intensity-modulated radiotherapy (PBMS-IMRT) with routine IMRT in patients undergoing radiochemotherapy for cervical cancer.

Material and methods: Forty patients with indications for adjuvant radiochemotherapy after cervical cancer surgery were selected and randomly divided into IMRT $(n=20)$ and PBMS-IMRT $(n=20)$ groups to observe and record the toxicity and its severity in the blood, gastrointestinal tract, and genitourinary system.

Results: There was no significant difference in the target area conformity index $(\mathrm{Cl})$ or homogeneity index $(\mathrm{HI})$ between the two groups $(p>0.05)$. The pelvic bone V10-V50 in the PBMS-IMRT group were lower than those in the IMRT group $(p<0.05)$, and there was lower hematological toxicity $(p<0.05)$ and fewer delays or interruptions in chemotherapy and/or radiotherapy $(p<0.05)$ in the PBMS-IMRT group. The toxicity to the gastrointestinal and genitourinary systems in the two groups was not significantly different $(p>0.05)$.

Conclusions: PBMS-IMRT significantly reduced the dose volume of the pelvic bone marrow, thereby reducing the incidence of bone marrow suppression. However, it had no significant impact on the gastrointestinal or genitourinary systems.

Key words: cervical cancer; confined pelvic bone marrow; intensity-modulated radiotherapy; toxicity
\end{abstract}

Ginekologia Polska 2023; 94, 2: 101-106

\section{INTRODUCTION}

Cervical cancer is the third most common malignant tumor in women worldwide, and it has always ranked first among gynecological malignancies in China. In recent years, cisplatin-based concurrent radiochemotherapy has become the standard treatment for advanced cervical cancer. Compared with radiotherapy alone, concurrent radiochemotherapy can reduce mortality by $30-50 \%[1-3]$; however, it is associated with increased toxicity, especially acute hematological toxicity that may cause Grade 3 or higher bone marrow suppression and force patients to stop radiochemotherapy $[4,5]$. Approximately $50 \%$ of adult bone marrow hematopoiesis is concentrated in the pelvic bone marrow and lower vertebral body [6, 7]. Several studies have shown that effectively reducing the volume of bone marrow irradiated during radiotherapy can reduce the risk of bone marrow suppression in patients with concurrent cervical cancer chemoradiotherapy [6-10]. Based on the above studies, as well as on the need to ensure precise coverage of the tumor target area while protecting organs at risk (OAR), this study clinically observed whether pelvic bone marrow-sparing intensity-modulated radiotherapy (PBMS-IMRT) can reduce acute side effects and ensure the smooth progress of concurrent radiochemotherapy in patients with cervical cancer.

\section{MATERIAL AND METHODS \\ General clinical information}

Forty patients who were admitted to our hospital for the first time after surgery for early cervical cancer between May 2016 and May 2017 were selected and divided into PBMS-IMRT $(n=20)$ and IMRT $(n=20)$ groups using the random table method. All selected patients had undergone extensive hysterectomy + pelvic lymph node dissection, were pathologically diagnosed with cervical cancer (squamous cell carcinoma or non-squamous cell carcinoma), and had no preoperative radiotherapy or chemotherapy. The indications for postoperative radiotherapy included: 
(1) having one high-risk factor after surgery (positive lymph node metastasis, positive surgical margin, or parauterine infiltration) and (2) having two medium-risk factors after surgery (tumor diameter $\geq 4 \mathrm{~cm}$, interstitial infiltration depth greater than one-third, lymphatic vascular interstitial infiltration, or adenocarcinoma). The postoperative stage was determined jointly by an associate chief physician or above from the Department of Oncology, the Department of Oncology, and the Department of Gynecologic Oncology. The exclusion criteria were as follows: (1) history of hypertension, diabetes, heart disease, liver disease, kidney disease, neurologic diseases, other serious diseases, other tumors, or had received radiotherapy or chemotherapy; (2) no routine blood tests or computed tomography (CT) examination before chemoradiotherapy, abnormal blood indicators before chemoradiotherapy, or distant metastasis on $\mathrm{CT}$; or (3) contraindications to radiotherapy and chemotherapy. The study was conducted in accordance with the principles of the Declaration of Helsinki. This study was approved by the ethics committee of Wenzhou Medical University. Written informed consent was obtained from all participants.

\section{Simulative positioning and target area outline}

Each patient was first placed in a fixed position using a vacuum air cushion and then enhanced CT scan positioning was performed using a Volume Zoom CT scanner (Smatom Series, Siemens Healthineers, Erlangen, Germany) with a scanning layer thickness of $5 \mathrm{~mm}$ and a scanning range of $\mathrm{L} 3-5 \mathrm{~cm}$ under the pubic symphysis. The images were then transmitted to the therapy planning system (TPS) (Pinnacle 9.10 Radiation Therapy Planning System, Philips Healthcare, Amsterdam, Netherlands) to outline the target area. Referring to the Delineation Guidelines issued by the Tumor Radiation Therapy Cooperative Organization (RTOG) [11], the clinical target volume (CTV) of the target area was delineated in the TPS system, including that of the common iliac lymph nodes, internal and external iliac lymph nodes, anterior sacral lymph nodes, obturator lymph nodes, lymphatic cysts (if any), surgical stump, and $3 \mathrm{~cm}$ of the proximal vagina; the area $5 \mathrm{~mm}$ exterior to the CTV ( $2 \mathrm{~mm}$ to the rectal side) was defined as the planning target volume (PTV). The CTVs of the small intestines, bladder, rectum, spinal cord, and bilateral femoral heads were also delineated. For the PBMS-IMRT group, the CTV of the pelvic bone (all hip bones, sacrococcyx, and upper femurs in the radiation field) was delineated, and the area $5 \mathrm{~mm}$ exterior to it was defined as the PTV. The clinical goals of the dose-volume of the OAR were $\mathrm{V} 30<38 \%$ for the small intestine, V $40<45 \%$ for the bladder, and V50 $<20 \%$ for the rectum. The clinical goals of the dose-volume of the OAR in the PBMS-IMRT group were $\mathrm{V} 20<76 \%$ and $\mathrm{V} 40<35 \%$ [11]. All 40 patients were treated as planned.

\section{Radiotherapy plan}

The treatment plans in the two groups were designed in the TPS system, the 7-field irradiation method was used with an X-ray energy of $6 \mathrm{MV}$, and a Varian 23EX medical linear accelerator (Varian Medical Systems, Palo Alto, CA) was used to implement the radiotherapy plan. The prescribed dose of the PTV was 50 Gy over 25 fractions for five weeks with a $95 \%$ isodose curve surrounding the PTV. Acceptable evaluation plans were assessed by clinicians and physicists based on clinical requirements.

\section{Dosimetric evaluation}

Combined evaluation was performed by clinicians and physicists using dose-volume histograms. The homogeneity index $(\mathrm{HI})$ and conformity index $(\mathrm{Cl})$ were as follows: $\mathrm{HI}=\mathrm{D} 5 / \mathrm{D} 95, \mathrm{Cl}=$ V95/PTV, where D5 represents the PTV dose of $5 \%$ of the target area, D95 represents the PTV dose of $95 \%$ of the target area, and V95 represents the exposure volume enclosed by a $95 \%$ isodose surface of the prescribed dose. The $\mathrm{HI}$ indicates the dose distribution in the target area; the smaller the value, the more uniform the dose distribution in the target area. The $\mathrm{Cl}$ indicates the consistency between the area surrounded by the isodose surface and the target area, in a range from 0 to 1 ; the larger the value, the better the fit.

\section{Chemotherapy}

Both the PBMS-IMRT and IMRT groups received concurrent chemotherapy during radiotherapy. The chemotherapy regimen consisted of weekly administration of cisplatin (CDDP) at $35-40 \mathrm{mg} / \mathrm{m}^{2}$ and radiotherapy on days $1,8,15$, 22,29 , and 36 .

\section{Classification criteria for acute radiation injury}

Acute radiation reactions occurred during treatment or within three months after the completion of radiotherapy. The American Radiotherapy Collaborative Group acute radiation injury classification standard [4] was adopted.

\section{Follow-up}

The follow-up period was August 2017. Each patient was followed up for at least three months, and the follow-up rate was $100 \%$. For each patient, blood tests were regularly performed in the clinic, and the patients were asked to self-report by telephone about radiation reactions in the digestive and genitourinary systems.

\section{Statistical analysis}

The statistical software package SPSS 20.0 (IBM, Armonk, NY) was used to analyze the data, and t-tests were used to compare the target area and the dose-volume parameters in OAR between the two groups. The corrected fourfold table $\chi^{2}$ test was used to compare the delay and/or interruption rates of 
chemotherapy and/or radiotherapy between the two groups. The non-parametric Mann-Whitney U test was used to compare acute reactions in the blood, digestive, and urinary systems. Statistical significance was established at $a=0.05$ and $p<0.05$.

\section{RESULTS}

The patients were aged between 30-62 years, with a median age of 50 years and Karnofsky Performance Status (KPS) [12] scores of $\geq 90$ points, including 12 cases of stage IB1, 9 cases of stage IB2, 8 cases of stage IIA1, and 11 cases of stage IIA2.

\section{Evaluation of the PTV target area coverage}

There was no significant difference in $\mathrm{Cl}$ or $\mathrm{HI}$ values between the two groups $(p>0.05)$ (Tab. 1).

\section{Comparison of the dose-volume parameters in OAR}

There was a significant difference in dose-volume parameters of the pelvis at various levels between the two

\begin{tabular}{|l|c|c|c|}
\multicolumn{4}{|c|}{ Table 1. Comparison of $\mathrm{Cl}$ and $\mathrm{HI}$ between groups $(\overline{\mathrm{x}} \pm \mathrm{s})$} \\
\hline Group & $\mathbf{n}$ & $\mathbf{C l}$ & $\mathbf{H I}$ \\
\hline PBMS-IMRT & 20 & $0.863 \pm 0.025$ & $0.103 \pm 0.024$ \\
\hline IMRT & 20 & $0.852 \pm 0.030$ & $0.093 \pm 0.015$ \\
\hline $\mathrm{t}$ & & 1.260 & 1.580 \\
\hline $\mathrm{p}$ & & 0.215 & 0.122 \\
\hline
\end{tabular}

$\mathrm{Cl}$ - conformity index; $\mathrm{HI}$ - homogeneity index; PBMS-IMRT — pelvic bone marrow-sparing intensity-modulated radiotherapy; IMRT — intensitymodulated radiotherapy groups, but not in the dose-volume parameters of the small intestine, bladder, or rectum between the two groups (Tab. 2).

\section{Comparison of toxicity}

The severity of hematological toxicity in the IMRT group was significantly higher than that in the PBMS-IMRT group $(Z=-2.186, p=0.038)$. There was no significant difference in the severity of toxicity in the lower digestive $\operatorname{tract}(Z=-1.492, p=0.136)$ or the urinary tract $(Z=-1.399$, $\mathrm{p}=0.162)($ Tab. 3-5).

\section{Impact on the completion of the chemotherapy and radiotherapy plans}

The PBMS-IMRT group had a significantly better delay rate and/or discontinuation rate of chemotherapy and/or radiotherapy than the IMRT group $(p<0.05)$ (Tab. 6).

\section{DISCUSSION}

Concurrent radiochemotherapy is mainly used in patients with stage IIB-IVA cervical cancer. Multiple randomized controlled studies have shown that concurrent radiochemotherapy can reduce the risk of death by $30-50 \%$ compared to radiotherapy alone. However, concurrent radiochemotherapy can also cause severe bone marrow suppression, thus delaying the completion of treatment plans for cervical cancer, or even causing the suspension of treatment plans in cases with severe bone marrow suppression. This negatively affects the prognosis. Therefore, to reduce toxicity in the blood and ensure completion of the planned

Table 2. Comparison of dose-volume parameters in the endangered organs $(\bar{X} \pm s)$

\begin{tabular}{|c|c|c|c|c|c|}
\hline Group & Dose-volume & PBMS-IMRT & IMRT & $\mathbf{t}$ & $p$ \\
\hline \multirow{5}{*}{ Pelvis } & $\mathrm{V}_{10}$ & $85.98 \pm 3.01$ & $90.07 \pm 2.83$ & -4.427 & $<0.001$ \\
\hline & $V_{20}$ & $72.43 \pm 4.98$ & $80.02 \pm 4.88$ & -4.868 & $<0.001$ \\
\hline & $\mathrm{V}_{30}$ & $52.91 \pm 4.34$ & $58.72 \pm 5.24$ & -3.819 & $<0.001$ \\
\hline & $V_{40}$ & $33.63 \pm 4.23$ & $38.12 \pm 5.97$ & -2.744 & 0.005 \\
\hline & $\mathrm{V}_{50}$ & $11.46 \pm 1.33$ & $16.21 \pm 3.22$ & -6.097 & $<0.001$ \\
\hline \multirow{5}{*}{ Small intestine } & V10 & $80.66 \pm 5.30$ & $82.67 \pm 5.12$ & -1.220 & 0.231 \\
\hline & V20 & $60.37 \pm 7.29$ & $64.59 \pm 8.00$ & -1.744 & 0.089 \\
\hline & V30 & $30.28 \pm 6.03$ & $30.96 \pm 5.27$ & -0.380 & 0.706 \\
\hline & V40 & $11.34 \pm 5.62$ & $14.00 \pm 5.94$ & -1.438 & 0.159 \\
\hline & V50 & $4.30 \pm 1.18$ & $4.27 \pm 1.36$ & 0.075 & 0.941 \\
\hline \multirow{4}{*}{ Bladder } & V20 & $100 \pm 0$ & $100 \pm 0$ & 0 & 1.000 \\
\hline & V30 & $76.43 \pm 8.32$ & $75.99 \pm 8.65$ & 0.164 & 0.871 \\
\hline & V40 & $42.33 \pm 7.32$ & $42.20 \pm 6.70$ & 0.059 & 0.954 \\
\hline & V50 & $15.29 \pm 2.02$ & $14.72 \pm 1.95$ & 0.908 & 0.370 \\
\hline \multirow{4}{*}{ Rectum } & V20 & $100 \pm 0$ & $100 \pm 0$ & 0 & 1.000 \\
\hline & V30 & $94.37 \pm 2.49$ & $94.11 \pm 2.63$ & 0.321 & 0.321 \\
\hline & V40 & $46.30 \pm 10.34$ & $45.29 \pm 8.62$ & 0.340 & 0.739 \\
\hline & V50 & $8.92 \pm 2.46$ & $8.99 \pm 2.50$ & -0.089 & 0.929 \\
\hline
\end{tabular}

PBMS-IMRT — pelvic bone marrow-sparing intensity-modulated radiotherapy; IMRT — intensity-modulated radiotherapy 
Table 3. Comparison of toxicity in the blood system between groups

\begin{tabular}{|l|c|c|c|c|c|}
\hline \multirow{2}{*}{ Group } & $\mathbf{n}$ & \multicolumn{4}{|c|}{ Hematological toxicity } \\
\cline { 3 - 6 } & & Level 0 & Level 1 & Level 2 & Level 3 \\
\hline PBMS-IMRT & 20 & 4 & 11 & 3 & 4 \\
\hline IMRT & 20 & 2 & 9 & 5 & \\
\hline Z & -2.186 & & & \\
\hline p & 0.038 & & & \\
\hline
\end{tabular}

PBMS-IMRT — pelvic bone marrow-sparing intensity-modulated radiotherapy; IMRT — intensity-modulated radiotherapy

Table 4. Comparison of toxicity in the lower digestive tract between groups

\begin{tabular}{|l|c|c|c|c|c|}
\hline Group & $\mathbf{n}$ & \multicolumn{3}{|c|}{ Toxicity in lower digestive tract } \\
\hline & & Level 0 & Level 1 & Level 2 & Level 3 \\
\hline PBMS-IMRT & 20 & 3 & 12 & 3 & 2 \\
\hline IMRT & 20 & 1 & 10 & 5 & \\
\hline Z & -1.492 & & & & \\
\hline p & 0.136 & & & \\
\hline
\end{tabular}

PBMS-IMRT — pelvic bone marrow-sparing intensity-modulated radiotherapy; IMRT — intensity-modulated radiotherapy

Table 5. Comparison of toxicity in the Urinary system between groups

\begin{tabular}{|l|c|c|c|c|c|}
\hline \multirow{2}{*}{ Group } & $\mathbf{n}$ & \multicolumn{4}{|c|}{ Toxicity in urinary system } \\
\cline { 2 - 5 } & & Level 0 & Level 1 & Level 2 \\
\hline PBMS-IMRT & 20 & 4 & 11 & 3 \\
\hline IMRT & 20 & 2 & 9 & 5 \\
\hline Z & -1.399 & & & \\
\hline i & 0.162 & & & \\
\hline
\end{tabular}

PBMS-IMRT — pelvic bone marrow-sparing intensity-modulated radiotherapy; IMRT — intensity-modulated radiotherapy

\begin{tabular}{|l|c|c|c|c|c|}
\hline \multicolumn{2}{|l|}{ Table 6. Comparison of delay or discontinuation rates of chemotherapy and/or radiotherapy between groups } \\
\hline Group & $\begin{array}{c}\text { Cases with delayed or discontinued } \\
\text { chemotherapy and/or radiotherapy }\end{array}$ & $\begin{array}{c}\text { Cases without delay or discontinuation rates of } \\
\text { chemotherapy and/or radiotherapy }\end{array}$ & $\begin{array}{c}\text { Sum } \\
\text { Delay or } \\
\text { discontinuation rate }\end{array}$ \\
\hline PBMS-IMRT & 1 & 19 & 12 & 20 \\
\hline IMRT & 8 & & $40 \%$ \\
\hline$X^{2}$ & 5.161 & & 20 \\
\hline P & 0.023 & & \\
\hline
\end{tabular}

PBMS-IMRT — pelvic bone marrow-sparing intensity-modulated radiotherapy; IMRT — intensity-modulated radiotherapy

concurrent radiochemotherapy, it is necessary to study how to reduce the irradiation volume of the hematopoietic bone marrow [4, 13-16].

More than $50 \%$ of the hematopoietic activity in the bone marrow is located in the lumbosacral spine, ilium, ischium, pubis, and proximal femurs, and these areas are exposed to varying degrees during pelvic radiotherapy for cervical cancer. Most studies have confirmed that myelosuppression in patients undergoing pelvic radiochemotherapy is related to the volume of the bone marrow receiving 10 or 20 Gy doses [6, 7]. Zhu et al. [17] analyzed 102 cervical can- cer patients receiving pelvic radiotherapy combined with cisplatin chemotherapy ( $40 \mathrm{mg} / \mathrm{m}^{2} /$ week) in three American centers, none of whom received granulocyte monocyte colony-stimulating factor (GM-CSF) or platelet transfusion therapy. Through the functional logarithmic transformation of time (weeks), they found that the weekly peripheral blood cell counts (In [white blood cells (WBCs)] and In [absolute neutrophil counts (ANC)]) were reduced and that there was a significant correlation between the increase in average photobiomodulation (PBM) radiation doses (V20, V30, and V40) and the weekly reduction of WBC and ANCs. With 
each 1-Gy increase in PBM, In (ANC) decreased by $9.6 / \mu \mathrm{L} /$ week (95\% confidence interval, 1.9-17.3, $\mathrm{p}=0.015$ ). Subgroup analysis revealed a significant association between weekly decreases in $\ln$ (WBC) and $\ln$ (ANC) among the lumbosacral spine, ischium, and proximal femur. Therefore, the incidence of acute blood toxicity can be decreased by reducing the dose of pelvic radiation. Three-dimensional chemoradiotherapy (3D-CRT) and IMRT are the two technologies currently used to treat pelvic cancer. IMRT reduces the radiation dose to normal pelvic tissues. Compared with 3D-CRT, although the volume of bone marrow exposed to IMRT is lower, the incidence of bone marrow suppression in patients with cervical cancer undergoing concurrent IMRT and radiochemotherapy is still high. To date, there is no consensus on the ability of IMRT to reduce blood toxicity compared with 3D-CRT technology [18]. At present, most pelvic IMRT radiotherapy plans do not limit pelvis-endangering doses in the radiation field, and physicists have not paid enough attention to further reduce the radiation dose-volume of the pelvis. Lujan et al. [19] proposed a dosimetric study of bone-limited pelvic IMRT (BMS-IMRT) and concluded that it could reduce bone marrow toxicity. Mell et al. [20] conducted a phase II study on bone marrow-sparing RT and Huang et al. [21] reported that PBMS-IMRT reduced the incidence of hematologic toxicity in patients with cervical cancer receiving concurrent chemoradiotherapy. A single-center prospective randomized controlled trial from 2020 is also underway.

In this study, 40 patients with early-stage cervical cancer were divided into IMRT and PBMS-IMRT groups. The comparison of dosimetry and toxicity in the blood, digestive, and urinary systems between the two groups revealed that the severity of hematological toxicity in the IMRT group was significantly higher than that in the PBMS-IMRT group, and the on-time completion of the radiotherapy plan in the IMRT group was significantly worse than that in the PBMS-IMRT group ( $p<0.05$ ). There were no significant differences in the severity of digestive and urinary system toxicities $(p>0.05)$. The hematological toxicity results of this study are similar to those of Lujan et al. [22] and Gandhi et al. [23], but the results of Mundt et al. [24] could be useful in indicating the toxicity reported in the other studies as numbers. However, this study had a short study period and a small sample size, and signs of radiotoxicity in the lower digestive tract and urogenital system can take months to years to appear [4]. The impact on subacute and chronic toxic reactions, as well as on long-term survival, requires further study.

In summary, PBMS-IMRT significantly reduced radiation exposure to the pelvic bone marrow and reduced the incidence of bone marrow suppression, thus ensuring on-time completion of radiochemotherapy plans. This not only expands the body of research but is also clinically significant.
The impact on subacute and chronic toxic reactions as well as on long-term survival requires further study.

\section{Conflicts of interest}

The authors declare no conflict of interest.

\section{REFERENCES}

1. Rose PG, Bundy BN, Watkins EB, et al. Concurrent cisplatin-based radiotherapy and chemotherapy for locally advanced cervical cancer. N Engl J Med. 1999; 340(15): 1144-1153, doi: 10.1056/NEJM199904153401502, indexed in Pubmed: 10202165.

2. Datta NR, Stutz E, Liu M, et al. Concurrent chemoradiotherapy vs. radiotherapy alone in locally advanced cervix cancer: A systematic review and meta-analysis. Gynecol Oncol. 2017; 145(2): 374-385, doi: 10.1016/j. ygyno.2017.01.033, indexed in Pubmed: 28188016.

3. Vordermark D. Radiotherapy of Cervical Cancer. Oncol Res Treat. 2016; 39(9): 516-520, doi: 10.1159/000448902, indexed in Pubmed: 27614991.

4. Kirwan JM, Symonds P, Green JA, et al. A systematic review of acute and late toxicity of concomitant chemoradiation for cervical cancer. Radiother Oncol. 2003; 68(3): 217-226, doi: 10.1016/s0167-8140(03)00197-x, indexed in Pubmed: 13129628.

5. Nicholas S, Chen L, Choflet A, et al. Pelvic Radiation and Normal Tissue Toxicity. Semin Radiat Oncol. 2017; 27(4): 358-369, doi: 10.1016/j.semradonc.2017.04.010, indexed in Pubmed: 28865519.

6. Mauch P, Constine L, Greenberger J, et al. Hematopoietic stem cell compartment: acute and late effects of radiation therapy and chemotherapy. Int J Radiat Oncol Biol Phys. 1995; 31(5): 1319-1339, doi: 10.1016/03603016(94)00430-S, indexed in Pubmed: 7713791.

7. Hui B, Zhang Y, Shi F, et al. Association between bone marrow dosimetric parameters and acute hematologic toxicity in cervical cancer patients undergoing concurrent chemoradiotherapy: comparison of three-dimensional conformal radiotherapy and intensity-modulated radiation therapy. Int J Gynecol Cancer. 2014; 24(9): 1648-1652, doi: 10.1097/IGC.0000000000000292, indexed in Pubmed: 25275663.

8. Bao Z, Wang D, Chen S, et al. Optimal dose limitation strategy for bone marrow sparing in intensity-modulated radiotherapy of cervical cancer. Radiat Oncol. 2019; 14(1): 118, doi: 10.1186/s13014-019-1324-y, indexed in Pubmed: 31378200.

9. Mell LK, Sirák I, Wei L, et al. INTERTECC Study Group. Bone marrow-sparing intensity modulated radiation therapy with concurrent cisplatin for stage IB-IVA cervical cancer: an international multicenter phase II clinical trial (INTERTECC-2). Int J Radiat Oncol Biol Phys. 2017; 97(3): 536-545, doi: 10.1016/j.jijobp.2016.11.027, indexed in Pubmed: 28126303.

10. Albuquerque K, Giangreco D, Morrison C, et al. Radiation-related predictors of hematologic toxicity after concurrent chemoradiation for cervical cancer and implications for bone marrow-sparing pelvic IMRT. Int J Radiat Oncol Biol Phys. 2011; 79(4): 1043-1047, doi: 10.1016/j. ijrobp.2009.12.025, indexed in Pubmed: 20471182.

11. Lim K, Small W, Portelance L, et al. Gyn IMRT Consortium. Consensus guidelines for delineation of clinical target volume for intensity-modulated pelvic radiotherapy for the definitive treatment of cervix cancer. Int J Radiat Oncol Biol Phys. 2011; 79(2): 348-355, doi: 10.1016/j. ijrobp.2009.10.075, indexed in Pubmed: 20472347.

12. Terret C, Albrand G, Moncenix G, et al. Karnofsky Performance Scale (KPS) or Physical Performance Test (PPT)? That is the question. Crit Rev Oncol Hematol. 2011; 77(2): 142-147, doi: 10.1016/j.critrevonc.2010.01.015, indexed in Pubmed: 20185330.

13. Green JA, Kirwan JM, Tierney JF, et al. Survival and recurrence after concomitant chemotherapy and radiotherapy for cancer of the uterine cervix: a systematic review and meta-analysis. Lancet. 2001; 358(9284): 781-786, doi: 10.1016/S0140-6736(01)05965-7, indexed in Pubmed: 11564482.

14. Chen SW, Liang JA, Hung YC, et al. Concurrent weekly cisplatin plus external beam radiotherapy and high-dose rate brachytherapy for advanced cervical cancer: a control cohort comparison with radiation alone on treatment outcome and complications. Int J Radiat Oncol Biol Phys. 2006; 66(5): 1370-1377, doi: 10.1016/j.jjrobp.2006.07.004, indexed in Pubmed: 16979836.

15. Phillips P, Phillips J. Hysterectomy with radiotherapy or chemotherapy or both for women with locally advanced Cervical Cancer. Clin Nurse Spec. 2017; 31(4): 189-190, doi: 10.1097/NUR.0000000000000304, indexed in Pubmed: 28594668. 
16. Kong $\mathrm{W}$, Wang $\mathrm{Y}$, Lv N, et al. Incidence of radiation enteritis in cervical cancer patients treated with definitive radiotherapy versus adjuvant radiotherapy. Journal of Cancer Research and Therapeutics. 2018; 14(8): 120, doi: 10.4103/0973-1482.163762.

17. Zhu He, Zakeri K, Vaida F, et al. Longitudinal study of acute haematologic toxicity in cervical cancer patients treated with chemoradiotherapy. J Med Imaging Radiat Oncol. 2015; 59(3): 386-93; quiz 394, doi: 10.1111/1754-9485.12297, indexed in Pubmed: 25828068.

18. Peng W, Zhao Y. Comparative of patients with cervical cancer using 3D-CRT and IMRT. 2016 9th International Congress on Image and Signal Processing, BioMedical Engineering and Informatics (CISP-BMEI). 2016, doi: 10.1109/cisp-bmei.2016.7853018.

19. Lujan AE, Mundt AJ, Yamada SD, et al. Intensity-modulated radiotherapy as a means of reducing dose to bone marrow in gynecologic patients receiving whole pelvic radiotherapy. Int J Radiat Oncol Biol Phys. 2003; 57(2): 516-521, doi: 10.1016/s0360-3016(03)00521-2, indexed in Pubmed: 12957265.

20. Mell LK, Sirák I, Wei L, et al. INTERTECC Study Group. Bone Marrow-sparing Intensity Modulated Radiation Therapy With Concurrent Cisplatin For Stage IB-IVA Cervical Cancer: An International Multicenter Phase II Clini- cal Trial (INTERTECC-2). Int J Radiat Oncol Biol Phys. 2017; 97(3): 536-545, doi: 10.1016/j.ijrobp.2016.11.027, indexed in Pubmed: 28126303.

21. Huang J, Gu F, Ji T, et al. Pelvic bone marrow sparing intensity modulated radiotherapy reduces the incidence of the hematologic toxicity of patients with cervical cancer receiving concurrent chemoradiotherapy: a single-center prospective randomized controlled trial. Radiat Oncol. 2020; 15(1): 180, doi: 10.1186/s13014-020-01606-3, indexed in Pubmed: 32727497.

22. Lujan AE, Mundt AJ, Yamada SD, et al. Intensity-modulated radiotherapy as a means of reducing dose to bone marrow in gynecologic patients receiving whole pelvic radiotherapy. Int J Radiat Oncol Biol Phys. 2003; 57(2):516-521, doi: 10.1016/s0360-3016(03)00521-2, indexed in Pubmed: 12957265.

23. Gandhi AK, Sharma DN, Rath GK, et al. Early clinical outcomes and toxicity of intensity modulated versus conventional pelvic radiation therapy for locally advanced cervix carcinoma: a prospective randomized study. Int J Radiat Oncol Biol Phys. 2013; 87(3): 542-548, doi: 10.1016/j. ijrobp.2013.06.2059, indexed in Pubmed: 24074927.

24. Mundt AJ, Roeske JC, Lujan AE, et al. Initial clinical experience with intensity-modulated whole-pelvis radiation therapy in women with gynecologic malignancies. Gynecol Oncol. 2001; 82(3): 456-463, doi: 10.1006/gyno.2001.6250, indexed in Pubmed: 11520140. 